\title{
A Study on the Optimal Portfolio Strategies Under Inflation
}

\author{
Mei YU \\ Research Center of Applied Finance, School of Banking and Finance, University of International \\ Business and Economics, Beijing 100029, China \\ E-mail: yumei@uibe.edu.cn \\ Qian GAO \\ Research Center of Applied Finance, School of Banking and Finance, University of International \\ Business and Economics, Beijing 100029, China \\ Zijian LIU \\ Research Center of Applied Finance, School of Banking and Finance, University of International \\ Business and Economics, Beijing 100029, China \\ Yike ZHOU \\ Research Center of Applied Finance, School of Banking and Finance, University of International \\ Business and Economics, Beijing 100029, China \\ Dan RALESCU \\ Department of Mathematical Sciences, University of Cincinnati, USA

\begin{abstract}
This paper tests the inflation hedging ability of four categories of important financial assets in China: Commodity futures, real estate, gold and industry stock and select the assets that have significant inflation hedging effect. Then the authors construct the mean-variance model under the inflation factor, using the selected assets to construct the inflation hedging portfolio, solving the model and obtain the optimal investment strategy with inflation protection function. The result shows that the portfolio constructed by the model have more stable real returns and its inflation hedging ability can be even better if the short selling restriction of stocks is eliminated.
\end{abstract}

Keywords inflation protection; mean-variance; investment portfolio

\section{Introduction}

For investors in financial markets, one of the most basic risks is the erosion of real return of the portfolio by inflation. As the rising price level shrinks the real value of assets, how to maintain and increase the property value under the inflation is closely related to everyone's economic interests. For a long time, Chinese economic growth excessively rely on the government investment, bank credit and the currency issuance which is lack of close monitoring mechanism,

Received July 31, 2014, accepted September 11, 2014

Supported by Humanity and Social Science Foundation of Ministry of Education of China (14YJA790075), "the Fundamental Research Funds for the Central Universities" in UIBE (15JQ04) 
the high-speed economic growth is always accompanied by the risk of inflation. Especially in recent five years, due to the successive outbreak of financial crisis and the European debt crisis, the United States, Japan and other countries continue to implement quantitative easing monetary policy, causing the excess of liquidity. At the same time, in order to cope with the international financial crisis, Chinese government put a large amount of currency into circulation. According to the data released by central bank, the total M2 in 2013 is 110.65 trillion Yuan, increased $13.6 \%$ compared with the same period of last year. The problem of excess liquidity is intensifying in the economic system as well as the possibility of severe inflation. In addition, the expectation of Ren's appreciation leads to a large number of hot money flow into China, exacerbates the inflation risk in our country. Inflation influences the foundation of the national economy and people's livelihood such as wages, prices, interest rates, investment and employment as well as related to economic and social stability. At the same time, inflation reduces the real purchasing power of assets held by investors, affects the investment return and shrinks real wealth of people. Therefore, under the background of currently rising price level, study the inflation hedging properties of the various assets and construct portfolio with inflation hedging functions becoming an important theoretical and practical issue that draws great attention among many scholars.

The research about the relation between asset returns and inflation started in the 1970s. Fisher ${ }^{[1]}$ has defined inflation as the situation of continuous significant rising of the general price level. Assets with inflation protection ability has the following properties: Their real rate of return are independent of the inflation rate, determined by factors like the productivity and risk of the assets as well as investors risk preference; their expected nominal rate of return changes with inflation simultaneously. Based on the hypothesis of Fisher, Fama and Schwert ${ }^{[2]}$ established a model to test the inflation protection ability. Hereafter, scholars abroad did a lot of work on the examination of inflation protection function among various assets.

Real estate is generally regarded as an ideal investment instruments to store value, most studies have shown that real estate investment have inflation protection function. Fama and Schwert's study based on the data of the US have concluded that private property is the only investment instrument that is able to hedge both expected and unexpected inflation. Based on their study, the subsequent scholars have done research mainly from the perspective of different time span and categories. In the aspect of stock, most studies come to conclusions that conflicted with the Fisher Effect, whether studies based on developed countries such as America, Britain, or studies based on emerging market economies like Hong Kong, Taiwan, Korea, Singapore etc. The related studies ${ }^{[3-5]}$ showed a negative correlation between stock returns and inflation rate. Most of empirical researches ${ }^{[6-7]}$ concluded that stocks can't provide effective hedge against inflation, but there are also studies ${ }^{[8-10]}$ have shown that in the long run, stock returns have not been eroded by inflation and there exists a time interval effect. Studies about gold find that in the short term, gold price is directly affected by factors such as the international monetary system, monetary policy, gold supply and demand and the war, consequently, gold is not a effective tool to hedge inflation in the short term. However, gold is good at storing value in the long run. Representative researches are [11-14].

Domestic scholars have also made a deep study of the relationship between inflation and 
asset returns. Studies about real estate investment in China have found that real estate investment is an effective hedge against inflation only when the return is in the medium level or above but not too high. When the real estate market is overheated or too cold, investors' loss may be further exacerbated, relative studies are [15-17]. Gold has better inflation protection function in the long run, especially when the inflation is in the accelerating stage, relative studies are [18-20]. Similar to foreign research results, most of the domestic scholars' research showed that there is a negative relationship between stock returns and inflation in China, related studies are $[21,22]$. Longer stock holding period (30 months and above) will not necessarily improve the inflation hedging effect, nevertheless, holding period between 12 to 24 months can hedge inflation risk as well as save transaction costs, specific studies are [23].

In the aspect of asset allocation strategy considered inflation risk, Qin $^{[24]}$ studied the optimal portfolio model under the influence of inflation and carried out numerical simulation. Wang ${ }^{[25]}$, studied asset allocation strategy to avoid inflation risk from view of long-term investment. $\mathrm{Bu}^{[26]}$ studied the method of constructing commodity futures market index in China and proposed thoughts about how to construct effective inflation hedging portfolio. Other scholars studied consumption and investment problems concerned with inflation, trying to build mathematical models that can well reflect the influence of inflation on market. Main achievements focused on asset allocation problem under stochastic interest rate and inflation, such as [27].

As we can see from the above analysis, although there are many domestic studies about inflation, but most of the research merely limited to discuss the hedging ability of financial asset, few scholars consider the method of the portfolio construction under inflation. Although Qin proposed a optimal portfolio model, the study has some deficiencies: The model only considers the influence of inflation on the risk-free asset, ignoring its influence on financial assets return, and also neglects the assessment of inflation hedging ability of existing assets in order to determine the asset pool, which is an important part of portfolio construction.

This paper focuses on constructing inflation hedging portfolio based on the assessment results of the inflation protection function of various assets. We discuss the efficient frontier of portfolio under the following three cases: Without consideration of inflation and with short selling restriction, with consideration of inflation and with short selling restriction, with consideration of inflation and without short selling restriction. Through analyzing and comparing the real return and risk in the three cases above, we propose reference and thoughts to further development of inflation hedging instruments in Chinese financial market.

The structure of the paper is as follows: The second part analyzes the inflation hedging ability of financial assets in China; the third part proposes the method to build an inflation hedging portfolio; the fourth part draws the conclusion.

\section{The Examination of the Inflation Hedging Ability of the Financial Asset in China}

\subsection{Model Construction}

In this paper, we test the inflation hedging ability of commodity futures, gold, real estate and industry stock. The model divides the nominal return into three parts: real return rate, 
expected inflation rate and unexpected inflation rate, as shown in the following formula:

$$
E\left(R_{t} \mid \Omega_{t-1}\right)=E\left(r_{t} \mid \Omega_{t-1}\right)+\beta E\left(\pi_{t} \mid \Omega_{t-1}\right)+\gamma\left[\pi_{t}-E\left(\pi_{t} \mid \Omega_{t-1}\right)\right]
$$

$E\left(R_{t} \mid \Omega_{t-1}\right), E\left(r_{t} \mid \Omega_{t-1}\right), E\left(\pi_{t} \mid \Omega_{t-1}\right)$ respectively represent for the nominal expected return, real expected return and expected inflation of time $t$ based on the information of time $t-1$. $\pi_{t}-$ $E\left(\pi_{t} \mid \Omega_{t-1}\right)$, namely the difference between inflation rate and expected inflation rate, represent for unexpected inflation rate.

According to Formula (1), we can get the regression model in order to test the inflation hedging ability of assets.

$$
R_{t}=\alpha+\beta E\left(\pi_{t} \mid \Omega_{t-1}\right)+\gamma\left[\pi_{t}-E\left(\pi_{t} \mid \Omega_{t-1}\right)\right]+\eta_{t}
$$

In regression model above, $R_{t}$ is the dependent variable while $E\left(\pi_{t} \mid \Omega_{t-1}\right), \pi_{t}-E\left(\pi_{t} \mid \Omega_{t-1}\right)$ are independent variables. $\alpha$ is the real return rate, $\beta$ and $\gamma$ are estimators, $\eta_{t}$ is random error term, $\beta, \gamma$ 's meaning are explained in the following table.

Table 1 The meanings of the parameters

\begin{tabular}{ccc}
\hline $\begin{array}{c}\text { Parameter } \\
\text { interval }\end{array}$ & $\begin{array}{c}\beta \text { (measuring the hedging ability } \\
\text { against expected inflation) }\end{array}$ & $\begin{array}{c}\gamma \text { (measuring the hedging ability } \\
\text { against unexpected inflation) }\end{array}$ \\
\hline$<0$ & Negative hedging & Negative hedging \\
0 & No hedging effect & No hedging effect \\
$(0,1)$ & Partially hedging & Partially hedging \\
1 & Completely hedging & Completely hedging \\
$>1$ & Excess hedging & Excess hedging \\
\hline$\beta=\gamma=1$ & The nominal return rate change with expected and unexpected inflation \\
& rate simultaneously, therefore has perfect inflation hedging ability \\
\hline
\end{tabular}

\subsection{Data}

In our analysis, the sample period is from January 2010 to March 2014 except for the real estate. Due to the lack of availability of reliable real estate price data, the sample interval of the real estate price index is from June 2011 to March 2014. All the data are monthly year-on-year logarithm yield.

Inflation rate is obtained from the monthly year-on-year CPI data released by the National Bureau of Statistics and take the logarithm form.

There are various methods to measure the expected inflation rate and generally they can be divided into two categories. One is through market survey or to find financial market indicators to get price expectations. Studies abroad often use the three-month Treasury bills' yield to maturity as the expected rate of inflation. The other kind of method is by setting up econometric model between inflation and other economic variables, using statistical projection value of inflation rate as expected inflation indicators. Commonly used econometric methods including blanchard-quan (BQ) method, hodrick-precscott (HP) filter method, Kalman filter method, and so on. 
This paper chooses the one period lagged one-year national bond's yield to maturity as a proxy indicator of the expected inflation rate. The yield to maturity of the national bond is obtained from the China Government Securities Depository Trust \& Clearing Co. Ltd. Our choice has several reasons: Firstly, the expected inflation rate is the market expectation about the current inflation level based on the macroeconomic fundamentals, and the yield to maturity on the national bond reflects nominal interest rate determined by factors like the macroeconomic fundamentals and the national economic policy. Moreover, the yield to maturity on the national bond is publicly available, so it is a reasonable choice for the proxy variable of expected inflation. Secondly, by observing the correlation between CPI and the yield to maturity of 6 months, 1,2,3 year national bond, we found that the correlation coefficient between CPI and one period lagged one year national bond yield to maturity is the highest during the sample period, reflecting strong relationship between the two variables, as it is shown in Table 2. Thirdly, we take granger causality test to further examine the relationship between the two variables. The ADF test shows that the CPI, the yield to maturity on national bond are first-order integration series, so we take the granger causality test on the first order difference of the series, and the results are shown in Table 3. According to Table 3, the null hypothesis that the one period lagged one year national bond yield to maturity is the granger cause of inflation cannot be rejected. That is to say, incorporating the yields information can improve the forecast accuracy of inflation.

Table 2 The correlation coefficient between current inflation rate and yield to maturity of national bonds

\begin{tabular}{ccccc}
\hline National bond maturity & 6 months & 1 year & 3 year & 5 year \\
\hline One period & 0.28 & 0.3 & 0.29 & 0.29 \\
Current period & 0.27 & 0.29 & 0.26 & 0.26 \\
One period ahead & 0.18 & 0.2 & 0.21 & 0.23 \\
\hline
\end{tabular}

Table 3 The Granger causality test of one period lagged one year national bond YTM and current period inflation rate

\begin{tabular}{|c|c|c|c|}
\hline Null hypothesis & Observations & F_value & P_value \\
\hline $\mathrm{YTM}_{t-1}$ does not Granger Cause $\pi_{t}$ & 50 & 3.64741 & 0.0348 \\
\hline$\pi_{t}$ does not Granger Cause $\mathrm{YTM}_{t-1}$ & 50 & 0.0894 & 0.9147 \\
\hline
\end{tabular}

In the aspect of commodity futures, we studied the following sixteen kinds of future contracts from the Shanghai, Dalian, Zhengzhou futures exchange: Soybeans No. 1, soybeans No. 2, yellow corn, LLDPE, soybean meal, palm oil, soybean oil, PVC, cathode copper, aluminum, zinc, gold, natural rubber, fuel oil, rebar and wire rod. We chose the settlement price namely the market volume weighted strike price of the contract to calculate their return. Considering that the CPI is monthly data, in order to match the data frequency, we selected the settlement price at the end of each month to calculate the one-year holding period returns. In addition, due to commodity futures can be short, they have more advantages over stocks. 
Real estate returns are calculated based on the housing price index in the first-tier cities, second-tier cities and third-tier cities, which are released by the China Index Academy. The gold return is obtained from the closing price of spot gold from Shanghai Gold Exchange. In the aspect of industry stocks, we selected the closing price of Hushen 300 industry Index at the end of each month to calculate the one-year holding period yield, the included industries are energy, raw materials, industry, optional consumption, the main consumption, medicine and health care, finance and real estate, information technology, the utility and the telecommunication service.

\subsection{Empirical Results}

\subsubsection{Stationary Test}

We take the ADF test on the 30 assets return series and the inflation rate, expected and unexpected inflation rate. The results are as following: stationary series $(I(0)$ series) included three kinds of commodity futures: LLDPE, cathode copper, aluminum; three kinds of industry stocks: optional consumption, real estate and finance, utilities as well as the return rate of the real estate. Only gold return is second order integration series $(I(2)$ series); all the other series are first order integration series $(I(1)$ series).

\subsubsection{Commodity Futures}

We adopt Formula (2) in Section 2.1 to test commodity futures' ability to hedge against inflation. Since some series are non-stationary, the residual series after regression existing serial correlation. We adopt the generalized difference method to adjust, adding lagged residuals of order $k$ in the regression, combine Formula (2) with Formula (3), until the serial correlation disappeared and the residual series become white noise. The modified results are shown in Table 4.

$$
\eta_{t}=\sum_{q=1}^{k} a_{q} \eta_{t}+v_{t}
$$

Table 4 The inflation hedging effect of commodity futures (Formula 2)

\begin{tabular}{lcccccccc}
\hline \multicolumn{2}{c}{\begin{tabular}{c} 
Commodity \\
\multicolumn{1}{c}{ futures }
\end{tabular}} & $\begin{array}{c}\text { Parameter } \\
\text { estimation }\end{array}$ & $\begin{array}{c}\text { Standard } \\
\text { error }\end{array}$ & T_value & P_value & $\begin{array}{c}\text { Adjusted } \\
\mathrm{R}^{2}\end{array}$ & $\begin{array}{c}\text { D.W. } \\
\text { statistics }\end{array}$ \\
\hline Soybeans & $\alpha$ & $13.5862^{* *}$ & 5.6826 & 2.3732 & 0.0219 & 0.2761 & 1 & 2.2668 \\
No. 1 & $\beta$ & $-4.6097^{* *}$ & 1.9009 & -2.425 & 0.0193 & & & \\
& $\gamma$ & $4.5689^{* * *}$ & 0.9967 & 4.584 & 0 & & & \\
\hline Soybeans & $\alpha$ & $6.5100^{*}$ & 3.8065 & 1.7102 & 0.094 & 0.3239 & 1 & 2.5692 \\
No. 2 & $\beta$ & -1.0426 & 1.2733 & -0.8188 & 0.4171 & & \\
& $\gamma$ & $4.6801^{* *}$ & 0.6676 & 7.01 & 0 & & \\
\hline Yellow & $\alpha$ & 2.0036 & 4.9373 & 0.4058 & 0.6868 & 0.4944 & 1 & 0.271 \\
corn & $\beta$ & 1.858 & 1.6516 & 1.125 & 0.2664 & & \\
& $\gamma$ & $3.9653^{* *}$ & 0.866 & 4.5791 & 0 & & \\
\hline
\end{tabular}




\begin{tabular}{|c|c|c|c|c|c|c|c|c|}
\hline Soybean & $\alpha$ & 10.3984 & 10.8157 & 0.9614 & 0.3414 & 0.5985 & 1 & 2.325 \\
\hline \multirow[t]{2}{*}{ meal } & $\beta$ & -5.0476 & 3.618 & -1.3951 & 0.1697 & & & \\
\hline & $\gamma$ & $5.0335^{* *}$ & 1.897 & 2.6534 & 0.0109 & & & \\
\hline \multirow[t]{3}{*}{ Palm oil } & $\alpha$ & 4.7513 & 9.6569 & 0.492 & 0.6251 & 0.1562 & 1 & 2.6831 \\
\hline & $\beta$ & -4.2814 & 3.3203 & -1.3254 & 0.1916 & & & \\
\hline & $\gamma$ & $12.6096^{* *}$ & 1.6938 & 7.4447 & 0 & & & \\
\hline \multirow{3}{*}{$\begin{array}{l}\text { Soybean } \\
\text { oil }\end{array}$} & $\alpha$ & -7.0176 & 7.6503 & -0.9173 & 0.3638 & 0.1103 & 1 & 2.5797 \\
\hline & $\beta$ & 3.3167 & 2.5591 & 1.296 & 0.2014 & & & \\
\hline & $\gamma$ & $6.9864^{* *}$ & 1.3418 & 5.2067 & 0 & & & \\
\hline \multirow[t]{3}{*}{ PVC } & $\alpha$ & -14.0292 & 9.1278 & -1.537 & 0.1316 & 0.4561 & 1 & 2.5182 \\
\hline & $\beta$ & 2.2852 & 2.9916 & 0.7639 & 0.4491 & & & \\
\hline & $\gamma$ & $-4.8180^{* * *}$ & 1.3456 & -3.5806 & 0.0009 & & & \\
\hline \multirow[t]{3}{*}{ Zinc } & $\alpha$ & 51.4188 & 10.8584 & 4.7354 & 0 & 0.6267 & 1 & 2.6148 \\
\hline & $\beta$ & -20.1215 & 3.6322 & -5.5397 & 0 & & & \\
\hline & $\gamma$ & $3.7914^{*}$ & 1.9045 & 1.9908 & 0.0525 & & & \\
\hline \multirow{3}{*}{$\begin{array}{l}\text { Natural } \\
\text { rubber }\end{array}$} & $\alpha$ & $30.0532^{*}$ & 17.7198 & 1.696 & 0.0966 & 0.34934 & 1 & 2.6687 \\
\hline & $\beta$ & $-10.0857^{*}$ & 5.9274 & -1.7015 & 0.0956 & & & \\
\hline & $\gamma$ & $11.3264^{* *}$ & 3.1079 & 3.6444 & 0.0007 & & & \\
\hline \multirow[t]{3}{*}{ Fuel oil } & $\alpha$ & $30.4422^{* * *}$ & 6.9885 & 4.3561 & 0.0001 & 0.4356 & 1 & 2.2057 \\
\hline & $\beta$ & $-9.3696^{* * *}$ & 2.3377 & -4.008 & 0.0002 & & & \\
\hline & $\gamma$ & $3.8755^{* * *}$ & 1.2257 & 3.1618 & 0.0028 & & & \\
\hline \multirow[t]{3}{*}{ Rebar } & $\alpha$ & -9.1612 & 8.7644 & -1.0453 & 0.3016 & 0.462 & 1 & 2.3182 \\
\hline & $\beta$ & 1.2344 & 2.89 & 0.4271 & 0.6714 & & & \\
\hline & $\gamma$ & $5.7556^{* * *}$ & 1.3882 & 4.146 & 0.0002 & & & \\
\hline \multirow[t]{3}{*}{ Wire rod } & $\alpha$ & -9.6946 & 8.7695 & -1.1055 & 0.275 & 0.3276 & 1 & 2.5814 \\
\hline & $\beta$ & 1.7846 & 2.8916 & 0.6171 & 0.5403 & & & \\
\hline & $\gamma$ & $6.1308^{* * *}$ & 1.389 & 4.4137 & 0.0001 & & & \\
\hline \multirow[t]{3}{*}{ LLDPE* $^{*}$} & $\alpha$ & -1.2163 & 1.7681 & -0.6918 & 0.4926 & 0.1235 & 0 & 1.8677 \\
\hline & $\beta$ & 10.3752 & 6.7951 & 1.5269 & 0.1338 & & & \\
\hline & $\gamma$ & $9.7232^{* * *}$ & 3.5177 & 2.7641 & 0.0082 & & & \\
\hline \multirow{3}{*}{$\begin{array}{l}\text { Cathode } \\
\text { copper* }\end{array}$} & $\alpha$ & 4.1719 & 3.143 & 1.3274 & 0.1911 & 0.1255 & 0 & 2.6013 \\
\hline & $\beta$ & 19.2348 & 12.1479 & 1.5834 & 0.1203 & & & \\
\hline & $\gamma$ & $17.3851^{* * *}$ & 6.2888 & 2.7645 & 0.0082 & & & \\
\hline \multirow[t]{3}{*}{ Aluminum* } & $\alpha$ & 1.5563 & 0.4131 & 1.1013 & 0.2766 & 0.1204 & 0 & 2.1204 \\
\hline & $\beta$ & 7.315 & 5.4618 & 1.3393 & 0.1872 & & & \\
\hline & $\gamma$ & -0.375 & 1.9897 & -0.1885 & 0.8514 & & & \\
\hline Gold** & $\alpha$ & -0.37503 & 1.989694 & -0.18849 & 0.8514 & 0.0331 & 1 & 3.0286 \\
\hline
\end{tabular}




$\begin{array}{lllll}\beta & 3.525852 & 7.599895 & 0.463934 & 0.645 \\ \gamma & -1.90873 & 4.198887 & -0.45458 & 0.6516\end{array}$

Note: In the commodity futures column, items marked with $*$ denotes $I(0)$ series, ** denotes $I(2)$ series, the rest are $I(1)$ series. In the parameter estimation column, $*$ denotes significant at $10 \%$ level, $* *$ denotes significant at the $5 \%$ level, $* * *$ denotes significant at $1 \%$ level. $K$ denotes the order of lagged residuals that added according to Formula (3).

Table 5 The inflation hedging effect of commodity futures (Formula 4)

\begin{tabular}{|c|c|c|c|c|c|c|c|c|}
\hline \multicolumn{2}{|c|}{$\begin{array}{c}\text { Commodity } \\
\text { futures }\end{array}$} & \multirow{2}{*}{$\begin{array}{l}\text { Parameter } \\
\text { estimation } \\
-1.2433\end{array}$} & \multirow{2}{*}{$\begin{array}{c}\begin{array}{c}\text { Standard } \\
\text { error }\end{array} \\
3.63\end{array}$} & \multirow{2}{*}{$\begin{array}{c}\text { T_value } \\
-0.3425\end{array}$} & \multirow{2}{*}{$\begin{array}{c}\text { P_value } \\
0.7335\end{array}$} & \multirow{2}{*}{$\begin{array}{c}\text { Adjusted } \\
\mathrm{R}^{2} \\
0.0393\end{array}$} & \multirow{2}{*}{$\begin{array}{l}k \\
0\end{array}$} & \multirow{2}{*}{$\begin{array}{c}\begin{array}{c}\text { D.W. } \\
\text { statistics }\end{array} \\
2.3467\end{array}$} \\
\hline Soybeans & $\beta$ & & & & & & & \\
\hline No.1 & $\gamma$ & $2.7759^{*}$ & 1.5291 & 1.8153 & 0.0996 & & & \\
\hline Soybeans & $\beta$ & 1.0805 & 2.8501 & 0.3791 & 0.7063 & -0.0264 & 0 & 2.5328 \\
\hline No. 2 & $\gamma$ & -0.5564 & 1.4874 & -0.3741 & 0.7101 & & & \\
\hline Yellow & $\beta$ & 0.0969 & 1.0846 & 0.0894 & 0.9292 & 0.2132 & 0 & 2.1953 \\
\hline corn & $\gamma$ & $7.7006^{* * *}$ & 2.0783 & 3.7053 & 0.0006 & & & \\
\hline Soybean & $\beta$ & 6.8634 & 5.0825 & 1.3504 & 0.1835 & 0.1167 & 0 & 2.1167 \\
\hline meal & $\gamma$ & $-4.7549^{*}$ & 2.6524 & -1.7927 & 0.0796 & & & \\
\hline \multirow[t]{2}{*}{ Palm oil } & $\beta$ & -0.243 & 2.0829 & -0.1167 & 0.9076 & 0.0105 & 0 & 1.6258 \\
\hline & $\gamma$ & $-6.5064^{*}$ & 3.3991 & -1.6302 & 0.0599 & & & \\
\hline Soybean & $\beta$ & -0.1121 & 3.3222 & -1.1893 & 0.2404 & 0.0246 & 0 & 2.0034 \\
\hline oil & $\gamma$ & 3.951 & 1.7338 & -0.0647 & 0.9487 & & & \\
\hline \multirow[t]{2}{*}{ PVC } & $\beta$ & 0.1124 & 2.0502 & 0.0548 & 0.9565 & 0.0593 & 0 & 2.1406 \\
\hline & $\gamma$ & $-7.2765^{* *}$ & 3.5422 & -2.0542 & 0.0372 & & & \\
\hline \multirow[t]{2}{*}{ Zinc } & $\beta$ & -0.5211 & 5.4178 & -0.0962 & 0.9238 & -0.0587 & 0 & 1.8944 \\
\hline & $\gamma$ & -1.0816 & 2.8274 & -0.3825 & 0.7038 & & & \\
\hline \multirow[t]{2}{*}{ Fuel oil } & $\beta$ & 0.3177 & 1.8165 & 0.1749 & 0.8619 & -0.0231 & 0 & 1.7032 \\
\hline & $\gamma$ & $4.8679^{* *}$ & 1.7332 & 2.8086 & 0.0487 & & & \\
\hline \multirow[t]{2}{*}{ Rebar } & $\beta$ & 3.6593 & 4.2588 & 0.8592 & 0.3949 & 0.0027 & 0 & 1.7634 \\
\hline & $\gamma$ & -1.8799 & 2.3671 & -0.7941 & 0.4314 & & & \\
\hline \multirow[t]{2}{*}{ Wire rod } & $\beta$ & -0.8251 & 2.2532 & -0.3662 & 0.716 & 0.0218 & 0 & 2.1477 \\
\hline & $\gamma$ & $5.7384^{* *}$ & 2.8331 & 2.0255 & 0.044 & & & \\
\hline
\end{tabular}

Note: In the commodity futures column, items marked with $*$ denotes $I(0)$ series, ** denotes $I(2)$ series, the rest is $I(1)$ series. In the Parameter estimation column, * denotes significant at $10 \%$ level, $* *$ denotes significant at the $5 \%$ level, $* * *$ denotes significant at $1 \%$ level. $K$ denotes the order of lagged residuals that added according to Formula (3). 
Because the most asset returns are $I(1)$ series, stationary series can be obtained after first order difference.

$$
\nabla R_{t}=\beta \nabla E\left(\pi_{t} \mid \Omega_{t-1}\right)+\gamma \nabla\left[\pi_{t}-E\left(\pi_{t} \mid \Omega_{t-1}\right)\right]+\eta_{t}
$$

The test results of $I(1)$ series using Formula (4) are as shown in Table 5. Comparing with Table 5, we can find that the conclusion are basically the same, only the parameters significance decreased and the results are more stable.

Combine the results in Tables 4 and 5, we can find that commodity futures has no obvious hedging effect against expected inflation, but have good properties to hedge against unexpected inflation. Specifically speaking, in the $1 \%$ level of significance, the $\gamma$ of yellow corn, LLDPE, cathode copper are significantly different from zero; in the $5 \%$ level of significance, the $\gamma$ of PVC, fuel oil, wire rod are significantly different from zero; in the $10 \%$ level of significance, the gamma of Soybeans No. 1, Soybean meal, Palm oil are significantly different from zero. Among them, the $\gamma$ of Palm oil, Soybean meal, PVC are negative, indicating a negative inflation hedging effect, the other six kinds of commodity futures have positive inflation hedge effect.

\subsubsection{Industry Stocks}

We took the same test of industry stock; results are shown in Tables 6 and 7. Similar with results of commodities futures, the estimated results with the first order differenced data are basically the same and more stable. Combined Table 6 and Table 7, we can find that industry stocks not only have unexpected inflation hedging properties, but also have obvious hedging effect against expected inflation. Specifically speaking, in the $5 \%$ level of significance, the $\beta$ of main consumption, telecommunications, utilities are significantly different from zero; in the $10 \%$ level of significance, optional consumptions $\beta$ is significantly different from zero. Moreover, the optional consumption has positive hedging effect against expected inflation while others have negative hedging effect against inflation. In the $5 \%$ level of significance, the $\gamma$ of energy is significantly different from zero, in the $5 \%$ level of significance, the $\gamma$ of raw materials, optional consumption, main consumption are significantly different from zero. In addition, the $\gamma$ of energy and raw materials are negative, indicating they have negative hedging effect against unexpected inflation, the remaining two assets have positive hedging effect against unexpected inflation.

\subsubsection{Gold and Real Estate}

Because the real estate return are stationary series, so we only use Formula (2) to analyze, the results are shown in Table 8. Generally speaking, real estate have a good positive hedge ef-

fect on expected inflation, which the first-tier and third-tier cities have $\beta$ in $1 \%$ level significance and the second-tier cities have $\beta$ in $5 \%$ level significance. 
Table 6 The inflation hedging effect of industry stocks (Formula 2)

\begin{tabular}{|c|c|c|c|c|c|c|c|c|}
\hline $\begin{array}{l}\text { Industry } \\
\text { stocks }\end{array}$ & & $\begin{array}{l}\text { Parameter } \\
\text { estimation }\end{array}$ & $\begin{array}{c}\text { Standard } \\
\text { error }\end{array}$ & T_value & P_value & $\begin{array}{c}\text { Adjusted } \\
\mathrm{R}^{2}\end{array}$ & $k$ & $\begin{array}{c}\text { D.W. } \\
\text { statistics }\end{array}$ \\
\hline \multirow[t]{3}{*}{ Energy } & $\alpha$ & -13.6248 & 12.2318 & -1.1139 & 0.2712 & 0.3602 & 1 & 0.6436 \\
\hline & $\beta$ & 1.9105 & 4.0534 & 0.4713 & 0.6397 & & & \\
\hline & $\gamma$ & $-10.2833^{* * *}$ & 2.0425 & -5.0297 & 0 & & & \\
\hline \multirow{3}{*}{$\begin{array}{l}\text { Raw } \\
\text { materials }\end{array}$} & $\alpha$ & -18.7372 & 13.9666 & -1.3416 & 0.1865 & 0.2577 & 1 & 0.5437 \\
\hline & $\beta$ & 0.2202 & 4.6283 & 0.0476 & 0.9623 & & & \\
\hline & $\gamma$ & $-9.6502^{* * *}$ & 2.3322 & -4.1378 & 0.0002 & & & \\
\hline \multirow[t]{3}{*}{ Industry } & $\alpha$ & $-23.0265^{*}$ & 13.5786 & -1.6958 & 0.0968 & 0.0557 & 1 & 0.3004 \\
\hline & $\beta$ & 3.3442 & 4.4997 & 0.7432 & 0.4612 & & & \\
\hline & $\gamma$ & $4.9312^{* *}$ & 2.2674 & 2.1748 & 0.0349 & & & \\
\hline \multirow{3}{*}{$\begin{array}{l}\text { Main } \\
\text { consumption }\end{array}$} & $\alpha$ & $15.2468^{*}$ & 8.5148 & 1.7906 & 0.0801 & 0.5439 & 1 & 0.7758 \\
\hline & $\beta$ & $5.7081^{* *}$ & 2.8216 & 2.023 & 0.049 & & & \\
\hline & $\gamma$ & $9.4004^{* * *}$ & 1.4218 & 6.6115 & 0 & & & \\
\hline \multirow{3}{*}{$\begin{array}{l}\text { Medicine \& } \\
\text { health care }\end{array}$} & $\alpha$ & $33.7684^{*}$ & 17.5339 & 1.9259 & 0.0604 & 0.0255 & 1 & 0.1873 \\
\hline & $\beta$ & -8.2066 & 5.8104 & -1.4124 & 0.1647 & & & \\
\hline & $\gamma$ & -4.179 & 2.9279 & -1.4273 & 0.1604 & & & \\
\hline \multirow{3}{*}{$\begin{array}{l}\text { Information } \\
\text { technology }\end{array}$} & $\alpha$ & 4.1854 & 20.5708 & 0.2035 & 0.8397 & 0.0248 & 1 & 0.1404 \\
\hline & $\beta$ & -0.3379 & 6.8168 & -0.0496 & 0.9607 & & & \\
\hline & $\gamma$ & -3.1262 & 3.435 & -0.9101 & 0.3676 & & & \\
\hline \multirow{3}{*}{$\begin{array}{l}\text { Telecommuni- } \\
\text { cation service }\end{array}$} & $\alpha$ & $-44.2384^{* * *}$ & 16.2553 & -2.7215 & 0.0092 & 0.0593 & 1 & 0.2427 \\
\hline & $\beta$ & $11.7894^{* *}$ & 5.3867 & 2.1886 & 0.0339 & & & \\
\hline & $\gamma$ & 2.5666 & 2.7144 & 0.9455 & 0.3494 & & & \\
\hline \multirow{3}{*}{$\begin{array}{l}\text { Optional } \\
\text { consumption } \\
*\end{array}$} & $\alpha$ & -0.5743 & 2.9058 & -0.1976 & 0.8442 & 0.0694 & 0 & 0.5002 \\
\hline & $\beta$ & $-19.3140^{*}$ & 11.0992 & -1.7401 & 0.0888 & & & \\
\hline & $\gamma$ & $11.4604^{*}$ & 6.1322 & 1.8689 & 0.0683 & & & \\
\hline \multirow{3}{*}{$\begin{array}{l}\text { Finance and } \\
\text { real estate* }\end{array}$} & $\alpha$ & $-5.9033^{* *}$ & 2.2644 & -2.607 & 0.0124 & 0.0075 & 0 & 0.5222 \\
\hline & $\beta$ & 10.3731 & 8.6493 & 1.1993 & 0.2368 & & & \\
\hline & $\gamma$ & -1.0273 & 4.7787 & -0.215 & 0.8308 & & & \\
\hline \multirow[t]{3}{*}{ Utility* } & $\alpha$ & $-12.6281^{* * *}$ & 1.3629 & -9.2658 & 0 & 0.1168 & 0 & 0.5317 \\
\hline & $\beta$ & $13.5718^{* *}$ & 5.2057 & 2.6071 & 0.0124 & & & \\
\hline & $\gamma$ & -1.6817 & 2.8761 & -0.58470 .5617 & & & & \\
\hline
\end{tabular}

Note: In the industry stocks column, items marked with $*$ denotes $I(0)$ series, the rest are $I(1)$ series. In the Parameter estimation column, * denotes significant at $10 \%$ level, ** denotes significant at the $5 \%$ level, $* * *$ denotes significant at $1 \%$ level. $K$ denotes the order of lagged residuals that added according to Formula (3). 
Table 7 The inflation hedging effect of industry stocks (Formula 4)

\begin{tabular}{|c|c|c|c|c|c|c|c|c|}
\hline $\begin{array}{c}\text { Industry } \\
\text { stocks }\end{array}$ & & $\begin{array}{l}\text { Parameter } \\
\text { estimation }\end{array}$ & $\begin{array}{c}\text { Standard } \\
\text { error }\end{array}$ & T_value & P_value & $\begin{array}{c}\text { Adjusted } \\
\mathrm{R}^{2}\end{array}$ & $\mathrm{k}$ & $\begin{array}{c}\text { D.W. } \\
\text { statistics }\end{array}$ \\
\hline \multirow[t]{2}{*}{ Energy } & $\beta$ & 7.3305 & 7.3813 & 0.9931 & 0.326 & 0.1608 & 0 & 1.2466 \\
\hline & $\gamma$ & $-5.8087^{* *}$ & 2.8459 & -2.0411 & 0.0413 & & & \\
\hline Raw & $\beta$ & 8.6089 & 7.9433 & 1.0838 & 0.2842 & 0.1443 & 0 & 1.4484 \\
\hline material & $\gamma$ & $-2.4872^{*}$ & 1.4103 & -1.7636 & 0.0576 & & & \\
\hline \multirow[t]{2}{*}{ Industry } & $\beta$ & 7.8382 & 5.9006 & 1.3284 & 0.1908 & 0.2383 & 0 & 1.2972 \\
\hline & $\gamma$ & 1.6242 & 3.278 & 0.4955 & 0.6227 & & & \\
\hline Main & $\beta$ & $2.8590 * *$ & 1.3904 & 2.0562 & 0.0481 & 0.17 & 0 & 0.8808 \\
\hline consumption & $\gamma$ & $5.3178^{*}$ & 2.8554 & 1.8623 & 0.0872 & & & \\
\hline Medicine \& & $\beta$ & 5.2167 & 5.8139 & 0.8973 & 0.3743 & 0.0478 & 0 & 0.8624 \\
\hline health care & $\gamma$ & -2.0761 & 3.2299 & -0.6428 & 0.5236 & & & \\
\hline Information & $\beta$ & 5.108 & 6.1931 & 0.8248 & 0.4138 & 0.0867 & 0 & 1.2083 \\
\hline technology & $\gamma$ & -2.6735 & 3.4405 & -0.7771 & 0.4412 & & & \\
\hline Telecommuni- & $\beta$ & $9.1449 * *$ & 4.4804 & 2.0411 & 0.0457 & 0.3193 & 0 & 1.3992 \\
\hline cation service & $\gamma$ & -2.4757 & 3.5253 & -0.7023 & 0.4861 & & & \\
\hline
\end{tabular}

Table 8 The inflation hedging effect of real estate (Formula 2)

\begin{tabular}{|c|c|c|c|c|c|c|c|c|}
\hline $\begin{array}{l}\text { Real } \\
\text { estate }\end{array}$ & & $\begin{array}{l}\text { Parameter } \\
\text { estimation }\end{array}$ & $\begin{array}{c}\text { Standard } \\
\text { error }\end{array}$ & T_value & P_value & $\begin{array}{c}\text { Adjusted } \\
\mathrm{R}^{2}\end{array}$ & $k$ & $\begin{array}{c}\text { D.W. } \\
\text { statistics }\end{array}$ \\
\hline \multirow{3}{*}{$\begin{array}{l}\text { First-tier } \\
\text { city }\end{array}$} & $\alpha$ & 0.1534 & 0.3331 & 0.4606 & 0.6488 & 0.9378 & 1 & 2.0334 \\
\hline & $\beta$ & $2.5363^{* * *}$ & 0.6413 & 3.9549 & 0.0005 & & & \\
\hline & $\gamma$ & 1.0904 & 1.2537 & 0.8698 & 0.3921 & & & \\
\hline \multirow{3}{*}{$\begin{array}{l}\text { Second-tier } \\
\text { city }\end{array}$} & $\alpha$ & 0.1103 & 0.3217 & 0.3428 & 0.7344 & 0.8546 & 1 & 1.9339 \\
\hline & $\beta$ & $2.8792^{* *}$ & 1.2285 & 2.3436 & 0.0267 & & & \\
\hline & $\gamma$ & -0.8733 & 0.6113 & -1.2814 & 0.211 & & & \\
\hline \multirow{3}{*}{$\begin{array}{l}\text { Third-tier } \\
\text { city }\end{array}$} & $\alpha$ & 0.2347 & 0.3874 & 0.6058 & 0.5497 & 0.7223 & 1 & 2.2584 \\
\hline & $\beta$ & $5.5064^{* * *}$ & 1.5773 & 3.4909 & 0.0017 & & & \\
\hline & $\gamma$ & 1.046 & 0.7406 & 1.4123 & 0.1693 & & & \\
\hline
\end{tabular}

Note: $K$ denotes the order of lagged residuals that added according to Formula (3).

Gold yield are one order integration series $(I(1))$, so we analyzed by Formula (2) and Formula (4) respectively. The results are shown in Table 9, under $10 \%$ level of the significance, gold has the positive hedging effect against expected inflation. 
Table 9 The inflation hedging effect of gold

\begin{tabular}{|c|c|c|c|c|c|c|c|c|}
\hline $\begin{array}{c}\text { Test } \\
\text { equation }\end{array}$ & & $\begin{array}{l}\text { Parameter } \\
\text { estimation }\end{array}$ & $\begin{array}{c}\text { Standard } \\
\text { error }\end{array}$ & T_value & P_value & $\begin{array}{l}\text { Adjusted } \\
\qquad \mathrm{R}^{2}\end{array}$ & $k$ & $\begin{array}{c}\text { D.W. } \\
\text { statistics }\end{array}$ \\
\hline \multirow[t]{3}{*}{ Formula 2} & $\alpha$ & $29.1748^{* * *}$ & 5.7525 & 5.0717 & 0 & 0.7926 & 1 & 1.9825 \\
\hline & $\beta$ & $-10.8277^{* * *}$ & 1.923 & -5.6307 & 0 & & & \\
\hline & $\gamma$ & $10.7574^{* * *}$ & 0.9748 & 11.0353 & 0 & & & \\
\hline \multirow[t]{2}{*}{ Formula 4} & $\beta$ & $3.4752^{*}$ & 1.9967 & 1.7405 & 0.0885 & 0.0232 & 0 & 2.3352 \\
\hline & $\gamma$ & -3.5202 & 3.9374 & -0.894 & 0.3759 & & & \\
\hline
\end{tabular}

Note: $K$ denotes the order of lagged residuals that added according to Formula (3).

\section{The Construction of Inflation Hedging Portfolio by Mean Variance Method}

\subsection{Portfolio with Short Selling Restriction}

Markowitz proposed the classic portfolio theory in 1952, portfolio optimization can be divided into the following four types:

1) given the expected return, minimize risk;

2) given the risk, maximize the expected return;

3) minimize risk without consideration of expected return;

4) maximize expected return without consideration of risk.

1) and 2) are dual problems and have the same solution. The purpose of this paper is to study the portfolio construction strategy under inflation, namely minimizing the portfolio's volatility caused by inflation thus ensure a stable real return. Therefore we choose the first form to analyze, and the model can be expressed as following:

$$
\begin{gathered}
\text { Model } \mathrm{P}) \quad \min \sigma_{p}^{2}=\sum_{i=1}^{n} x_{i}^{2} \sigma_{i}^{2}+\sum_{i=1}^{n} \sum_{\substack{j=1 \\
j \neq i}}^{n} x_{i} x_{j} \sigma_{i j} \\
\text { s.t. } E\left(R_{p}\right)=\sum_{i=1}^{n} x_{i} \cdot E\left(R_{i}\right)=X^{\mathrm{T}} \cdot R \\
\sum_{i=1}^{n} x_{i}=1
\end{gathered}
$$

$E\left(R_{p}\right)$ represents expected return of the portfolio, $x_{i}$ represents the allocation weight of asset $i, X=\left(x_{1}, x_{2}, \cdots, x_{n}\right)^{\mathrm{T}}$ represents the weight vector of the portfolio of assets, $R=$ $\left(E\left(R_{1}\right), E\left(R_{2}\right), \cdots, E\left(R_{N}\right)\right)^{\mathrm{T}}$ represents the expected return vector of each asset, $\sigma_{p}^{2}$ represents the portfolio variance, $\sigma_{i}^{2}$ represents the variance of asset $i, \Sigma$ represents the variance covariance matrix of the portfolio's asset. 
In the classical Markowitz portfolio theory, asset returns are nominal returns, ignoring the influence of inflation and the related investment risk. This paper analyses how to construct the optimal portfolio under inflation, so the classic model need to be modified.

Considering the influence of inflation, the real return of the portfolio need to be taken into account, we define as asset inflation rate after the removal of $\mathrm{I}$, then when inflation is taken into account, we need to focus on the real return of the assets, thus we define $R_{i}^{\prime}$ as the real return after the elimination of inflation rate.

$$
E\left(R_{i}^{\prime}\right)=E\left(R_{i}\right)-\pi
$$

Here $\pi$ represents the inflation rate. We use $E\left(R_{i}^{\prime}\right)$ to denote the portfolio's expected return adjusted by inflation, which can be written as

$$
E\left(R_{i}^{\prime}\right)=\sum_{i=1}^{n}\left(x_{i} \cdot\left(E\left(R_{i}^{\prime}\right)\right)\right)=X^{\mathrm{T}} \cdot R_{\pi}
$$

In the formula above,

$$
X=\left(x_{1}, x_{2}, \cdots, x_{n}\right)^{\mathrm{T}}, \quad R_{\pi}=\left(E\left(R_{1}^{\prime}\right)-\pi, E\left(R_{2}^{\prime}\right)-\pi, \cdots, E\left(R_{N}^{\prime}\right)-\pi\right)^{\mathrm{T}}
$$

The portfolio variance with consideration of inflation can be expressed as

$$
\begin{aligned}
\sigma_{p}^{\prime 2} & =\operatorname{cov}\left(\sum x_{i} R_{i}-\pi, \sum x_{j} R_{j}-\pi\right) \\
& =\operatorname{cov}\left(\sum x_{i}\left(R_{i}-\pi\right), \sum x_{j}\left(R_{j}-\pi\right)\right) \\
& =\sum \sum x_{i} x_{j} \operatorname{cov}\left(R_{i}-\pi, R_{j}-\pi\right) \\
& =X^{\mathrm{T}} \sum_{\pi} X
\end{aligned}
$$

The $\sum_{\pi}$ in Formula (8) represents the variance covariance matrix after the inflation deduction asset be added into the portfolio. Now we get the optimal portfolio model with consideration of inflation risk.

$$
\begin{aligned}
(\text { Model Q) } \min & \sigma_{p}^{\prime 2}=X^{\mathrm{T}} \cdot \sum_{\pi} \cdot X \\
\text { s.t. } & E\left(R_{p}^{\prime}\right)=X^{\mathrm{T}} \cdot R_{\pi} \\
& \sum_{i=1}^{n} x_{i}=1
\end{aligned}
$$

2) The portfolio considered inflation risk and under the short sell restriction of stocks. First, let's review the test results in Part 2: Among the 30 kinds of asset of commodity futures, industry stocks, gold and real estate, gold, real estate and some industry stocks have obvious hedging effect against expected inflation while other industry stocks and commodity futures have nice hedging property against unexpected inflation. The remaining 10 asset returns have no significant relationship with inflation thus don't have the ability to hedge against inflation. Therefore, we eliminate those 10 assets in the process of portfolio construction, using the 20 kinds of assets left to construct the portfolio. The asset categories and the corresponding hedge property are shown in Table 10. 
Table 10 A brief summary of the inflation hedging effect of the tested assets

\begin{tabular}{|c|c|c|c|}
\hline Hedging against expected inflation & \multicolumn{3}{|c|}{ Hedging against unexpected inflation } \\
\hline Optional consumption & \multicolumn{3}{|l|}{ Energy } \\
\hline Main consumption & \multicolumn{3}{|l|}{ Raw materials } \\
\hline Telecommunication & \multicolumn{3}{|c|}{ Optional consumption } \\
\hline Utility & \multicolumn{3}{|c|}{ Main consumption } \\
\hline Gold & Soybeans No.1 & PVC & \\
\hline The real estate of first-tier cities & Yellow corn & LLDPE & \\
\hline \multirow[t]{2}{*}{ second-tier cities and third-tier cities } & Soybean meal & Palm oil & \\
\hline & Cathode copper & Fuel oil & Wire rod \\
\hline
\end{tabular}

In order to demonstrate the hedging effect of various asset more clearly, we add the 20 assets into the portfolio in three times. The first asset pool only contains six industry stocks and we denote the portfolio constructed base on the first asset pool as portfolio A; the second asset pool added gold and real estate on the basis of the first pool, and we denote the portfolio constructed base on the second pool as portfolio B. Considered gold and real estate can hedge against expected inflation, adding those assets will enhance the portfolio's ability to hedge against expected inflation; the third asset pool further add commodity futures which can hedge against unexpected inflation into the pool and we denote the portfolio constructed base on the third pool as portfolio C.

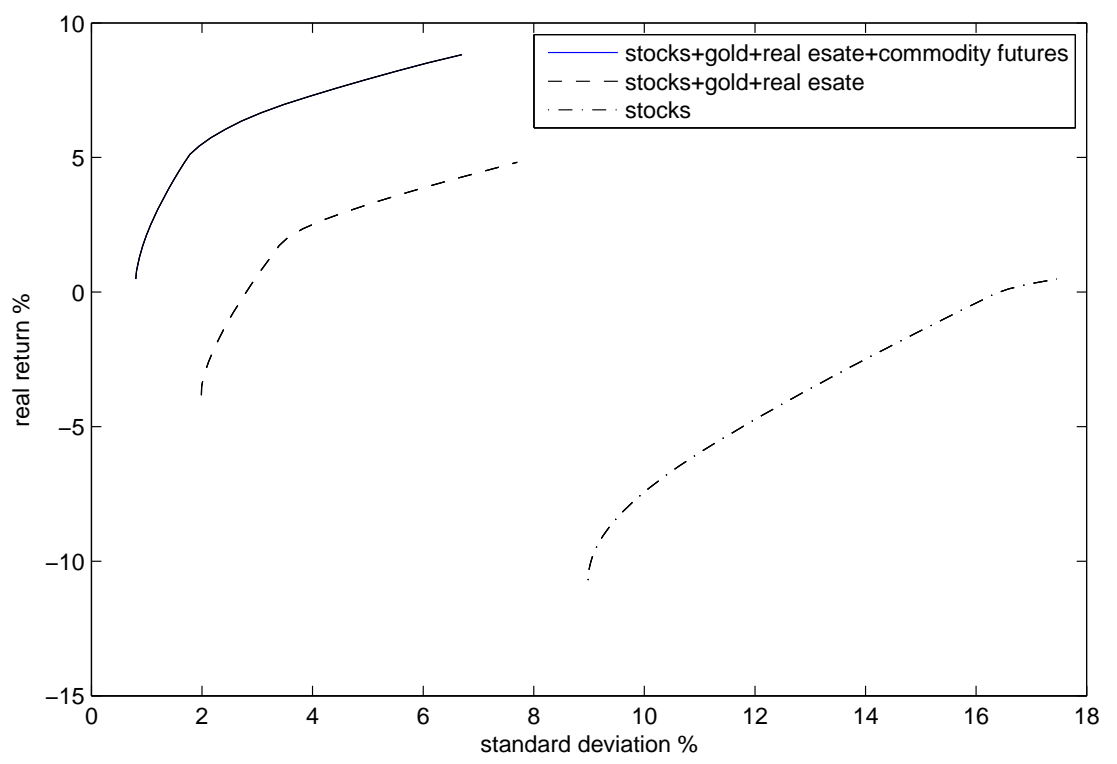

Figure 1 The portfolio efficient frontier inflation risk under short selling restriction

Because there is short selling restriction of stock and real estate in Chinese market, in order to conform with the reality, we add nonnegative constraints $x_{i} \geq 0$ to the seven industry stocks and three kinds of real estate assets in the solving process of model Q. Comparing 
portfolio A and B in Figure 1, we can find that the efficient frontiers move to the northwest obviously after gold and real estate added into the asset pool. This means that compared with portfolio A, portfolio B has more desirable properties: Given the same expected real return, risk is decreased; given the same risk, expected real return increased. Because the portfolio B mainly contains assets that can hedge against expected inflation, after commodity futures which can hedge against unexpected inflation joined into the pool, the efficient frontier move to the northwest further, which means portfolio $\mathrm{C}$ has more stable real returns.

Table 11 The asset allocation of the inflation hedging portfolio (with short selling restriction of stocks)

\begin{tabular}{|c|c|c|c|c|c|c|c|}
\hline \multicolumn{2}{|c|}{ A stocks } & \multicolumn{2}{|c|}{ B stocks+gold+real estate } & \multicolumn{4}{|c|}{ C stocks + gold +real estate+commodity futures } \\
\hline Name & Weight & Name & Weight & Name & Weight & Name & Weight \\
\hline Energy & 0 & Energy & 0 & Energy & 0 & Soybean No.1 & $9.80 \%$ \\
\hline Raw & 0 & Raw & 0 & Raw & 0 & Yellow corn & 0 \\
\hline materials & & materials & & materials & & & \\
\hline Optional & 0 & Optional & 0 & Optional & 0 & LLDPE & 0 \\
\hline consumption & & consumption & & consumption & & & \\
\hline Main & $29.61 \%$ & Main & 0 & Main & 0 & Soybean meal & $-5.76 \%$ \\
\hline consumption & & consumption & & consumption & & & \\
\hline Telecommun- & 0 & Telecommun- & 0 & Telecommun- & 0 & Palm oil & $-3.27 \%$ \\
\hline ication & & ication & & ication & & & \\
\hline \multirow[t]{9}{*}{ utility } & $70.39 \%$ & utility & $21.02 \%$ & utility & $27.30 \%$ & $\mathrm{PVC}$ & 0 \\
\hline & & Gold & $6.47 \%$ & Gold & $1.67 \%$ & Cathode & 0 \\
\hline & & & & & & copper & \\
\hline & & First-tier & 0 & First-tier & 0 & Fuel oil & $0.84 \%$ \\
\hline & & cities & & cities & & & \\
\hline & & Second-tier & 0 & Second-tier & 0 & Wire rod & 0 \\
\hline & & cities & & cities & & & \\
\hline & & Third-tier & $72.51 \%$ & Third-tier & $69.42 \%$ & & \\
\hline & & cities & & cities & & & \\
\hline Standard & $8.98 \%$ & & $1.99 \%$ & & $0.80 \%$ & & \\
\hline Deviation & & & & & & & \\
\hline Real return & $-10.70 \%$ & & $-3.83 \%$ & & $0.48 \%$ & & \\
\hline $\begin{array}{c}\text { Nominal } \\
\text { return }\end{array}$ & $-7.30 \%$ & & $-0.68 \%$ & & $3.63 \%$ & & \\
\hline
\end{tabular}

The asset allocation of the minimum variance portfolio is shown in Table 11. As we can see, because the stocks can't sell short, some stocks' weight can only be zero even if they have negative inflation hedging function. Only those with positive hedging function are endowed 
with weights. If short-selling are allowed, the stocks with negative inflation hedging function will be able to really play their role in the portfolio and the portfolio efficient frontier could further move to the northwest. While the asset category increased, the risk of minimum variance portfolio gradually decreased, the standard deviation are $8.98 \%, 1.99 \%, 0.80 \%$ respectively; the real return gradually increased, the real return rate are $-10.70 \%,-3.83 \%, 0.48 \%$ respectively.

With the asset allocation of portfolio $\mathrm{C}$ in Table 11, combined with the asset returns during the sample period, we can get the return series of portfolio $\mathrm{C}$. The ADF test shows that the return of portfolio $\mathrm{C}$ is first-order integration series $(I(1))$, so we adopt Formulas (2) and (4) to test its inflation protection function accordingly. The results are shown in Table 12: The test result by Formula (2) shows that both $\beta$ and $\gamma$ are significantly different from zero under $1 \%$ significance level; the test using Formula (4) shows that $\gamma$ is significantly different from zero under $10 \%$ significance level, indicating the portfolio have positive hedging function against inflation.

Table 12 The inflation hedging effect of portfolio C (Considered inflation risk and under short selling restriction)

\begin{tabular}{lcccccccc}
\hline $\begin{array}{c}\text { Test } \\
\text { equation }\end{array}$ & $\begin{array}{c}\text { Parameter } \\
\text { estimation }\end{array}$ & $\begin{array}{c}\text { Standard } \\
\text { error }\end{array}$ & T_value & P_value & $\begin{array}{c}\text { Adjusted } \\
\mathrm{R}^{2}\end{array}$ & $\begin{array}{c}\text { D.W. } \\
\text { statistics }\end{array}$ \\
\hline Formula 2 & $\alpha$ & $-11.1768^{* * *}$ & 1.3646 & -8.1904 & 0 & & & \\
& $\beta$ & $3.4439^{* * *}$ & 0.4343 & 7.9301 & 0 & 0.7900 & 1 & 1.9000 \\
& $\gamma$ & $1.4725^{* * *}$ & 0.1725 & 8.5389 & 0 & & & \\
\hline Formula 4 & $\beta$ & 1.4246 & 0.8571 & 1.6621 & 0.1073 & 0.1692 & 0 & 3.3732 \\
& $\gamma$ & $0.7752^{*}$ & 0.4251 & 1.8235 & 0.0786 & & & \\
\hline
\end{tabular}

3) The portfolio without consideration of inflation risk and under the short selling restriction of stocks

If we do not consider the inflation risk, we will not eliminate the asset that have no obvious correlation with inflation and use the nominal returns instead of real returns to solve model $\mathrm{P}$ in Section 1. For comparison, we get the real return of the portfolio by subtracting the average inflation rate during the simple period from the nominal return obtained by solving model $\mathrm{P}$. Then we plot the efficient frontier as shown in Figure 2. We also compare the efficient frontier of portfolio $\mathrm{C}$ with and without consideration of inflation risk in Figure 3. Comparing the upper part of efficient frontier with positive real return in Figure 3, we can clearly see that the portfolio considered the inflation risk are better, since it has lower risk under the same expected real return.

According to the ADF test, the returns of portfolio $\mathrm{C}$ are first-order integration series $(I(1))$, so we adopt the same method above to test its inflation hedging ability and the results are shown in Table 13. We can see that neither $\beta$ nor $\gamma$ are significantly different from zero, therefore portfolio $\mathrm{C}$ constructed without consideration of inflation risk indeed have no obvious inflation hedging effect and its return is more vulnerable to inflation volatility. 


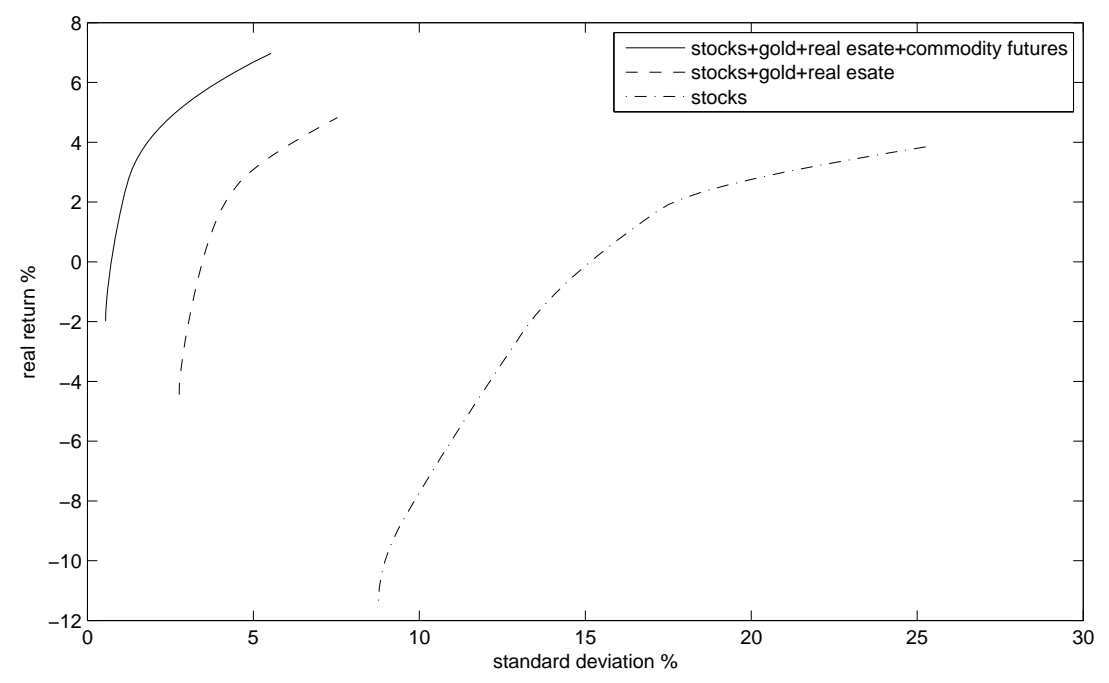

Figure 2 The portfolio efficient frontier without consideration of inflation risk under short selling restriction

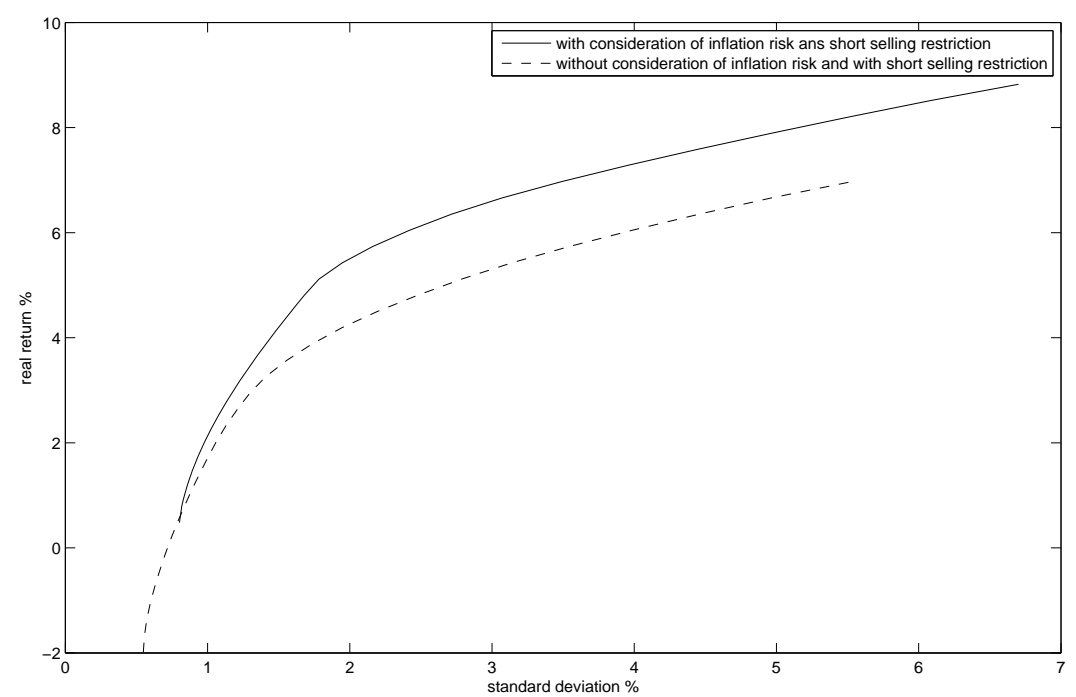

Figure 3 The comparison of efficient frontier under different conditions

Table 13 The inflation hedging effect of portfolio C (without considered inflation risk and under short selling restriction)

\begin{tabular}{lcccccccc}
\hline $\begin{array}{c}\text { Test } \\
\text { equation }\end{array}$ & $\begin{array}{c}\text { Parameter } \\
\text { estimation }\end{array}$ & $\begin{array}{c}\text { Standard } \\
\text { error }\end{array}$ & T_value & P_value & $\begin{array}{c}\text { Adjusted } \\
\mathrm{R}^{2}\end{array}$ & $\begin{array}{c}\text { D.W. } \\
\text { statistics }\end{array}$ \\
\hline Formula 2 & $\alpha$ & $-12.0791^{* * *}$ & 1.7595 & -6.8652 & 0 & & & \\
& $\beta$ & 3.4874 & 2.8394 & 1.2282 & 0.2705 & 0.5943 & 1 & 1.3359
\end{tabular}




\begin{tabular}{lcccccccc}
\multicolumn{1}{c}{$\gamma$} & 0.8467 & 1.1084 & 0.7639 & 0.4491 & & & \\
\hline Formula 4 & $\beta$ & 1.5284 & 1.0618 & 0.4394 & 0.1607 & 0.2015 & 0 & 1.0644 \\
& $\gamma$ & -1.1452 & 2.6811 & -0.4271 & 0.6714 & & & \\
\hline
\end{tabular}

\subsection{The Portfolio Without Short Selling Restriction of Stocks}

We once mentioned in the previous section that if short selling of stocks are allowed, then stocks with negative inflation hedging ability can play their role thus further increase the stability of portfolio's the real returns. In this section, we make empirical analysis on this assumption.

In the solving process of model $\mathrm{Q}$, we remove the nonnegative constraints of stocks and obtain the efficient frontier shown in Figure 4. The asset allocation of the minimum variance portfolio are shown in Table 14.

From Figure 4 we can see that, as the category of assets increased, the efficient frontier of portfolio A, B, C move to the northwest gradually. It means that given the same expected real return, the real return of portfolio $\mathrm{C}$ is more stable. Observing the returns and risk of the minimum variance portfolio in Table 14, we can get the same conclusion: As category of assets increased, the risk of portfolio is decreased, from $6.36 \%$ to $0.94 \%$ to $0.59 \%$; the real rate of return increased, from $-10.15 \%$ to $-1.36 \%$ to $2.35 \%$.

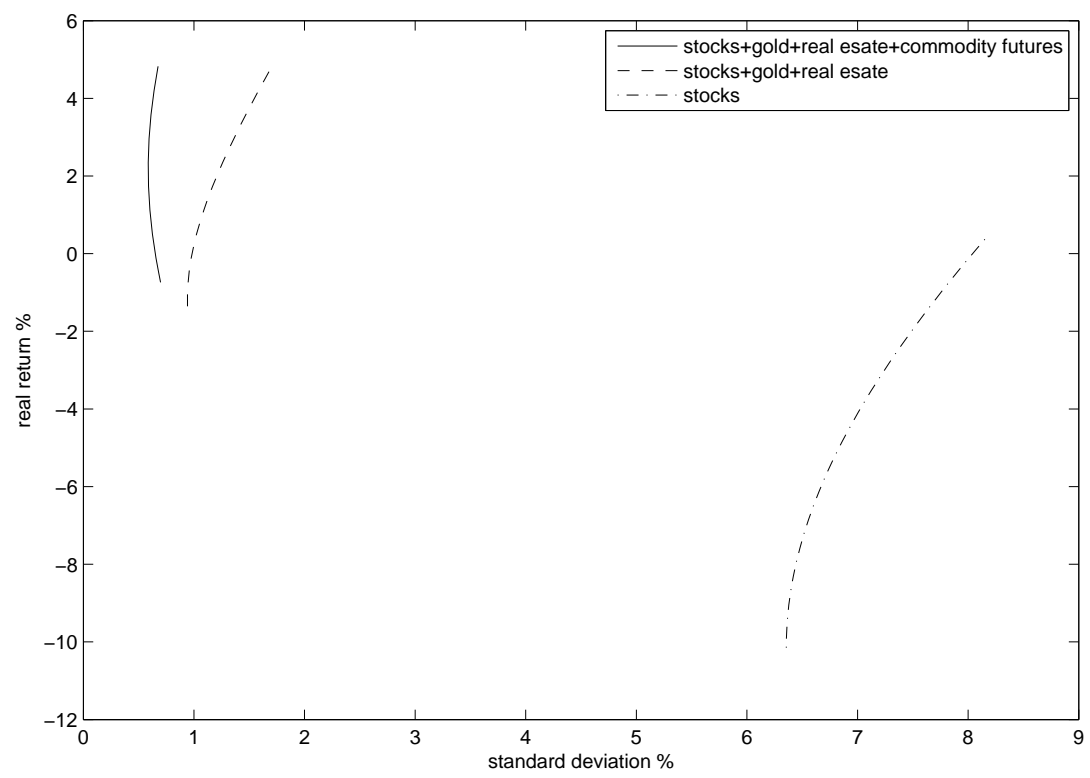

Figure 4 The portfolio efficient frontier with consideration of inflation risk and without short selling restriction

A further investigation of Table 14 we can find that the weight of stocks which have negative inflation hedging effect such as the energy, optional consumption and telecommunications etc, have changed from zero to negative, thus those stocks can play their role of hedging against 
inflation. In Figure 5, we compared the efficient frontier of portfolio $\mathrm{C}$ under the three cases we analyzed above: Without consideration of inflation risk and with short selling restriction of stocks, with consideration of inflation risk and short selling restriction of stocks, with consideration of inflation risk and without short selling restriction of stocks. We can see from Figure 5 that when short selling of stocks is allowed, the efficient frontier further move to the northwest, meaning the real return is more stable under the same expected real return, which supported the previous assumption we made.

Table 14 The asset allocation of the inflation hedging portfolio (without short selling restriction of stocks)

\begin{tabular}{|c|c|c|c|c|c|c|c|}
\hline \multicolumn{2}{|c|}{ A stocks } & \multicolumn{2}{|c|}{ B stocks +gold +real estate } & \multicolumn{4}{|c|}{ C stocks+gold +real estate+commodity futures } \\
\hline Name & Weight & Name & Weight & Name & Weight & Name & Weight \\
\hline Energy & $-21.44 \%$ & Energy & $1.64 \%$ & Energy & $-4.32 \%$ & Soybean No.1 & $7.03 \%$ \\
\hline $\begin{array}{c}\text { Raw } \\
\text { materials }\end{array}$ & $-4.39 \%$ & $\begin{array}{c}\text { Raw } \\
\text { materials }\end{array}$ & $-3.15 \%$ & $\begin{array}{c}\text { Raw } \\
\text { materials }\end{array}$ & $7.22 \%$ & Yellow corn & $26.95 \%$ \\
\hline $\begin{array}{c}\text { Optional } \\
\text { consumption }\end{array}$ & $-46.01 \%$ & $\begin{array}{c}\text { Optional } \\
\text { consumption }\end{array}$ & $5.93 \%$ & $\begin{array}{c}\text { Optional } \\
\text { consumption }\end{array}$ & $-3.30 \%$ & LLDPE & $3.81 \%$ \\
\hline $\begin{array}{c}\text { Main } \\
\text { consumption }\end{array}$ & $60.40 \%$ & $\begin{array}{c}\text { Main } \\
\text { consumption }\end{array}$ & $0.00 \%$ & $\begin{array}{c}\text { Main } \\
\text { consumption }\end{array}$ & $6.76 \%$ & Soybean meal & $-6.98 \%$ \\
\hline $\begin{array}{l}\text { Telecommun- } \\
\text { ication }\end{array}$ & $19.64 \%$ & $\begin{array}{l}\text { Telecommun- } \\
\text { ication }\end{array}$ & $-11.54 \%$ & $\begin{array}{l}\text { Telecommun- } \\
\text { ication }\end{array}$ & $-9.88 \%$ & Palm oil & $-4.94 \%$ \\
\hline \multirow[t]{5}{*}{ utility } & $91.81 \%$ & utility & $17.68 \%$ & utility & $16.59 \%$ & PVC & $-15.57 \%$ \\
\hline & & Gold & $-4.00 \%$ & Gold & $-6.63 \%$ & $\begin{array}{l}\text { Cathode } \\
\text { copper }\end{array}$ & $-2.26 \%$ \\
\hline & & $\begin{array}{l}\text { First-tier } \\
\text { cities }\end{array}$ & $8.78 \%$ & $\begin{array}{c}\text { First-tier } \\
\text { cities }\end{array}$ & $3.33 \%$ & Fuel oil & $8.47 \%$ \\
\hline & & $\begin{array}{l}\text { Second-tier } \\
\text { cities }\end{array}$ & $-45.31 \%$ & $\begin{array}{l}\text { Second-tier } \\
\text { cities }\end{array}$ & $-2.04 \%$ & Wire rod & $-8.42 \%$ \\
\hline & & $\begin{array}{c}\text { Third-tier } \\
\text { cities }\end{array}$ & $129.97 \%$ & $\begin{array}{c}\text { Third-tier } \\
\text { cities }\end{array}$ & $84.19 \%$ & & \\
\hline $\begin{array}{l}\text { Standard } \\
\text { Deviation }\end{array}$ & $6.36 \%$ & & $0.94 \%$ & & $0.59 \%$ & & \\
\hline Real return & $-10.15 \%$ & & $-1.36 \%$ & & $2.35 \%$ & & \\
\hline $\begin{array}{c}\text { Nominal } \\
\text { return }\end{array}$ & $-6.75 \%$ & & $1.79 \%$ & & $5.50 \%$ & & \\
\hline
\end{tabular}

Similar to the analysis above, we investigate the inflation hedging ability of portfolio C. According to the ADF test, the return series of portfolio $\mathrm{C}$ is first-order integration series $(I(1))$, so we adopt Formula (2) and Formula (4) to test its inflation hedging ability. The 
results are shown in Table 15. The test result using Formula (2) have shown that both $\beta$ and $\gamma$ are different from zero under $1 \%$ significance level; using Formula (4) to test, the significance of $\beta$ decreased slightly, $\beta$ is different from zero under $5 \%$ significance level while $\gamma$ is different from zero under $1 \%$ significance level. In conclusion, the portfolio has positive hedging ability against inflation.

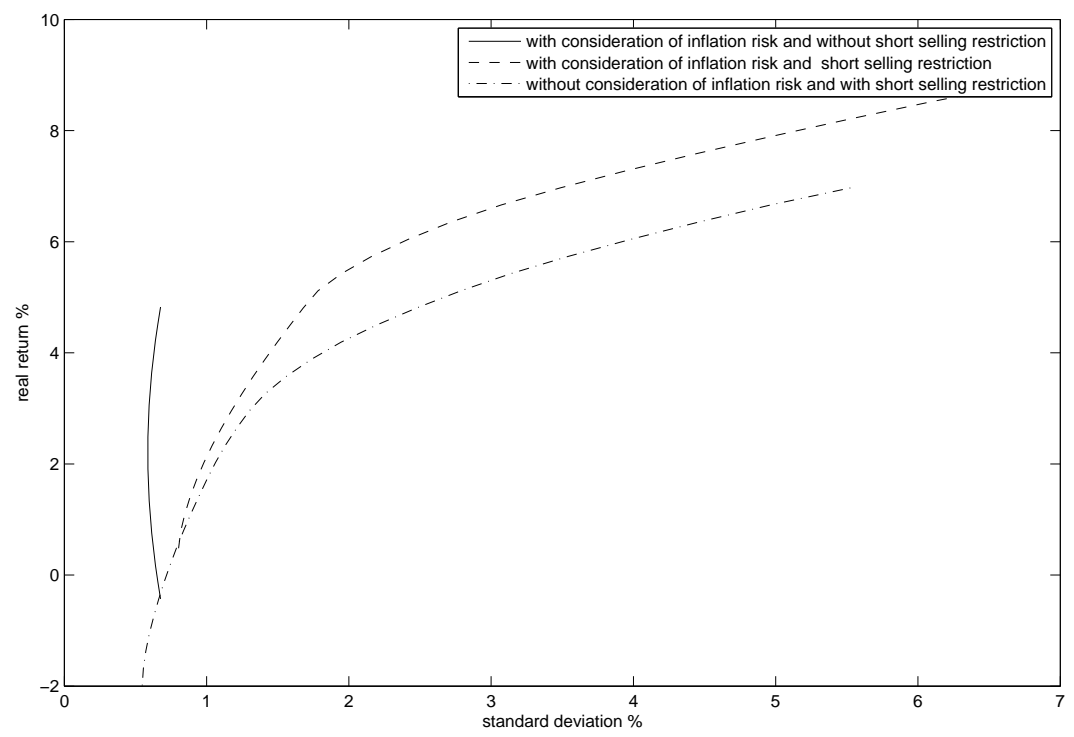

Figure 5 The comparison of efficient under different conditions

Table 15 The inflation hedging effect of portfolio $\mathrm{C}$ (with considered inflation risk and without short selling restriction of stocks)

\begin{tabular}{lcccccccc}
\hline $\begin{array}{c}\text { Test } \\
\text { equation }\end{array}$ & & $\begin{array}{c}\text { Parameter } \\
\text { estimation }\end{array}$ & $\begin{array}{c}\text { Standard } \\
\text { error }\end{array}$ & T_value & P_value & $\begin{array}{c}\text { Adjusted } \\
\mathrm{R}^{2}\end{array}$ & $\begin{array}{c}\text { D.W. } \\
\text { statistics }\end{array}$ \\
\hline Formula 2 & $\alpha$ & $1.9925^{* * *}$ & 0.6040 & 3.2989 & 0.0026 & & & \\
& $\beta$ & $1.1969^{* * *}$ & 0.1922 & 6.2269 & 0 & 0.8590 & 1 & 1.8006 \\
& $\gamma$ & $1.0003^{* * *}$ & 0.0763 & 13.1054 & 0 & & & \\
\hline Formula 4 & $\beta$ & $1.0634^{* *}$ & 0.5130 & 2.0728 & 0.0472 & 0.2231 & 0 & 3.3468 \\
& $\gamma$ & $0.7755^{* * *}$ & 0.2545 & 3.0473 & 0.005 & 0.2231 & 0 & 3.3468 \\
\hline
\end{tabular}

\section{Conclusions}

In this paper, we first test the inflation hedging ability of the following four categories of assets: Commodity futures, industry stocks, real estate and gold. The empirical results have shown that: Among the 16 kinds of commodity futures we tested, the following nine kinds of commodity futures have hedging ability against unexpected inflation: Soybeans No.1, yellow corn, LLDPE, soybean meal, palm oil, PVC, cathode copper, fuel oil and wire rod. The 
real estate of the first-tier, second-tier and third-tier cities and gold have desirable hedging ability against expected inflation; among the 10 kinds of industry stocks we tested, the optional consumption, the main consumption, utilities and telecommunications have hedging ability against expected inflation while the optional consumption, the main consumption, energy and raw materials have hedging ability against unexpected inflation.

Then, we constructed the mean variance model with consideration of inflation, using the 20 assets with obvious inflation hedging ability selected by the test results before to construct the optimal portfolio under inflation and obtain the efficient frontier. We compare the efficient frontier under three cases: Without consideration of inflation risk and with short selling restriction of stocks, with consideration of inflation risk and short selling restriction of stocks, with consideration of inflation risk and without short selling restriction of stocks, and we find that the efficient frontier moving to the northwest gradually. It means that from the first case to the third case, given the same return, the risk of the portfolio is decreasing thus the stability of the real return is increasing.

Finally, we test the inflation hedging ability of the minimum variance portfolio in the three cases above, and we find that the minimum variance portfolio constructed with consideration of inflation risk have obvious inflation hedging property and the hedging effect become more significant when the short selling of stocks is allowed. As currently the stocks are not allowed for short selling in our country, the portfolio construction strategy is limited to a certain degree. If the short selling restriction is eliminated in the future; the portfolio construction strategy in this paper can provide more scientific and accurate advice for the investors.

\section{References}

[1] Fisher I. The theory of interest. New York, Mad Millan, 1930, 6(2): 56-61.

[2] Fama E F, Schwert G W. Asset returns and inflation. Journal of Financial Economics, 1977, 5(2): 115-116.

[3] Bodie Z. Common stocks as a hedge against inflation. The Journal of Finance, 1976, 31(2): 459-470.

[4] Gultekin M N, Gultekin N B. Stock market seasonality: International evidence. Journal of Financial Economics, 1983, 12(4): 469-481.

[5] Adrangi B, Chatrath A, Dhanda K K, et al. Chaos in oil prices? Evidence from futures markets. Energy Economics, 2001, 23(4): 405-425.

[6] Liu C H, Hartzell D J, Hoesli M E. International evidence on real estate securities as an inflation hedge. Real Estate Economics, 1997, 25(2): 193-221.

[7] Gallagher L A, Taylor M P. The stock return-inflation puzzle revisited. Economics Letters, 2002, 75(2): $147-156$.

[8] Siegel J J. Stocks for the long run: The definitive guide to financial market returns and long-term investment strategies. New York: McGraw-Hill, 1998.

[9] Rapach D E. The long-run relationship between inflation and real stock prices. Journal of Macroeconomics, 2002, 24(3): 331-351.

[10] Engsted T, Tanggaard C. The relation between asset returns and inflation at short and long horizons. Journal of International Financial Markets, Institutions and Money, 2002, 12(2): 101-118.

[11] Moore G H. Gold prices and a leading index of inflation. Challenge, 1990, 33(4): 52-56.

[12] Ghosh D, Levin E J, Macmillan P, et al. Gold as an inflation hedge? University of St. Andrews, 2000.

[13] Ranson D, Wainwright H C. Inflation protection: Why gold works better than linkers. World Gold Council, 2005.

[14] Levin E J, Wright R E. Short run and long run determinants of the price of gold. The world Gold Council, 2006. 
[15] Zhang H, Zhang H. Empirical study of the relationship between inflation and housing prices. JournalTsinghua University, 2008, 48(3): 329-332.

[16] Glascock J L, Feng L, Fan L, et al. Inflation hedging characteristics of real estate assets in Hong Kong. SSRN Working Paper, 2008: 1180658.

[17] Di J P. Can real estate provide a hedge against inflation? - Evidence from Chinese mainland. China Real Estate, 2012(2): 10-17.

[18] Liu S, Hu Z. An analysis the stability of long-run determinants of the gold price. World Economy Study, 2008(2): 35-41.

[19] Yang N, He J Y. The analysis of changes in anti-inflation function of gold and corresponding determinants. Journal of Shanghai University of Finance and Economics, 2011, 13(6): 80-88.

[20] Nian S W. Can gold investment fight inflation? - Comments on the development of commercial bank's gold business. Shanghai Finance, 2011(3): 89-93.

[21] Liu J, Wang F. A positive analysis of the dynamic relationship between stock returns and inflation. The Study of Finance and Economics, 2004, 30(1): 123-128.

[22] Xiao C. An empirical analysis on the relationship between stock returns and inflation. Pioneering with Science \& Technology Monthly, 2006(5): 36-37.

[23] Wang Y Y, Li B, Zhang J P. On time section effect of the capacity of the stock market sterilizing inflation risk in China. West China Finance, 2012(4): 44-50.

[24] Qin S, Qin C Y, Chen R X. The optimal portfolios model with inflation rate. Systems Engineering Theory Methodology Application, 2004, 13(4): 316-319.

[25] Wang C F, Wu Q Q, Li H H, et al. How to manage inflation and stochastic interest rate risk in a strategic asset allocation: An investment problem with medium/long period. Systems Engineering, 2006, 24(4): 60-64.

[26] Bu H, Wang S Y. Empirical study of inflation-hedging characteristics of commodity futures and its portfolio in China. Journal of Management Sciences in China, 2010, 13(9): 26-36.

[27] LI H, Wang C, Wu Q. On the asset allocation problem under inflation, short and long stochastic interest rates risks. Journal of Beijing Institute of Technology (Social Sciences Edition), 2005(6): 30-33. 